\title{
COULD VIBRATIONAL SPECTROSCOPY REVOLUTIONISE QUALITY CONTROL OF HERBAL MEDICINES?
}

\section{(C) Viljoen Alvaro, Vermaak Ilze}

Department of Pharmaceutical Sciences, Faculty of Science, Tshwane University of Technology, Private Bag X680, Pretoria 0001, South Africa

Vibrational spectroscopy includes near infrared (NIR) mid-infrared (MIR) and Raman spectroscopy. These methods in combination with multivariate data analysis have proved to be powerful analytical techniques and have been implemented in various industries such as the petrochemical, agricultural and food industries. The quality of herbal medicines is of great concern as many consumers now choose herbal medicines over Western medicines and cases of mistaken identity (substitutions) or adulteration with other species with sometimes dire health consequences or loss of consumer trust in the product due to ineffectiveness has been reported. The quality of herbal medicines is assessed both qualitatively (authentication) and quantitatively through the quantification of specific biomarkers. Traditional analytical methods used to determine quality such as high performance liquid chromatography and mass spectrometry are destructive, expensive and time-consuming. Vibrational spec-

troscopy methods have major advantages over these methods in that it is simple, rapid, relatively cheap and non-destructive. Various examples of quality control methods developed using vibrational spectroscopy techniques will be described for medicinally and commercially important plant species: to discriminate between closely related plant species (e.g. Agathosma betulina and $A$. crenulata; Pelargonium reniforme and $P$. sidoides), a case of mistaken identity (Eriocephalus punctulatus and E. tenuifolius) and as a quantification tool for biomarkers (e. g. pulegone, diosphenol, isomenthone etc. in A. betulina essential oils). Hyperspectral imaging uses the platform of vibrational spectroscopy and multivariate data analysis but adds an extra data dimension opening up a range of novel applications in the quality control of herbal medicines such as authentication using whole dried fruits (e. g. Illicium anisatum and $I$. verum). From these examples it is evident, the revolution has already begun.

\section{THE METABOLISM OF LIGNANS FROM FRUCTUS SCHISANDRA}

\section{() Yin Jun ${ }^{1}$, Cao Yun-Feng ${ }^{1}$, Yang Ling ${ }^{2}$}

'School of Traditional Chinese Medicines, Shenyang Pharmaceutical University, Shenyang 110016, China ${ }^{2}$ Laboratory of Pharmaceutical Resource Discovery, Dalian Institute of Chemical Physics, Chinese Academy of Sciences, Dalian 116023, China

After metabolites from dibenzocyclooctadiene lignans (Schizandrin, Deoxyschizandrin and schisantherin A) of Schisandra chinensis incubated with human liver microsomes were isolated by HPLC and their structures were identified by $\mathrm{LC}-\mathrm{MS},{ }^{1} \mathrm{H}-\mathrm{NMR}$ and ${ }^{13} \mathrm{C}-\mathrm{NMR}$, the study combined correlation analysis, chemical inhibition studies, assays with recombinant CYPs and enzyme kinetics indicated that CYP3A4 was the main hepatic isoform. Three metabolites were isolated by HPLC and their structures were identified to be $8(R)$-hydroxylschizandrin, 2-demethyl-8 (R)-hydroxyl-schizandrin, 3-demethyl-8 (R)-hydroxyl-schizandrin, Rat and minipig liver microsomes were applied to evaluate species differences, and the results showed little difference among the mentioned species. Speculated that deoxyschizandrin might undergo hydroxylation in HLMs, and schizandrin could represent the corresponding metabolite. The data also show that Deoxyschizandrin metabolism to form schizandrin specially mediated by CYP3A4, is the principal route of disposition in humans. The specificity, high affinity, and high turnover make Deoxyschizandrin hydroxylation an excellent probe drug for CYP3A4 activity in vitro. The metabolic pathway of schisantherin $A$ validated the inhibitory mechanism against $C Y$ P3A4 undergoes demethylenenation in the human liver microsomes. $I_{50}$ drift and dynamics test indicate that schisantherin $A$ is inhibitor which shows dependency to NADP- and time, which illustrates that the methylene of schisantherin A produced active intermediate during the catalyzed reaction, thereby presents forceful inhibitory effect against CYP3A4. The lignans containing methylenedioxy abundant in Fructus Schisandra probably reduce clinical value thereof. In mice liver microsomes, schisantherin A, schizandrol B and schizandrin B showed strong inhibition against metablism schizandrin and deoxschizandrin. Our research results indicate that why deoxyschizandrin presented forceful anti-multidrug resistance in vitro but reduced or vanished in vivo, and the extract of Fructus Schisandra containing deoxyschizandrin presents forceful anti-multidrug resistance in vivo. 